\title{
Morphology Vendor Name
}

National Cancer Institute

\section{Source}

National Cancer Institute. Morphology Vendor Name. NCI Thesaurus. Code C117619.

The literal identifier of the vendor or laboratory that performs a morphology examination. 